\title{
La turbomacchina solare nelle applicazioni spaziali $(*)$
}

\author{
C. D'Anelio
}

Ricevuto il 19 Febbraio 1963

Rrassunto. - Fra i vari sistemi di conversione di energia per applicazioni spaziali la turbomacchina solare a vapore presenta uno dei campi di applicazione piǹ vasto nei riguardi della potenza.

Nel presente lavoro vengono esaminati taluni fra i principali problemi inerenti a tale tipo di macchina, con particolare riguardo alla scelta del ciclo termico e del fluido operante. Per quest'ultimo vengono indicate quelle che sono le caratteristiche ideali.

Sumarr. - Among the various systems of converting energy for space applications, the steam solar turbine-machine is one of the widest application fields with regard to power.

This paper examines some of the most important problems related to this kind of machine, with special reference to the choice of the thermal cycle and of the operating fluid. The ideal characteristics of the latter are also given.

\section{INTRODIZLONE.}

J'arvento ed il mapidissimo arescente sviluppo dei reicoli spaziali, siano essi orbitali o no, ha reso di importanza sempre maggiore lo sviluppo dello studio di generatori di potenza nello spazio.

I sisteni che possono essere adoperati, per tale scopo, sono diversi differenziandosi principalnente per il fatto di sfruttare energia presente nello spazio sotto forma raggiante (ad esempio energia solare) e trasformarla in energia direttamente ntilizzabile quale quella mecoanica od elettrica, o di trasportare da terra le fonti stesse dellenergia, sotto forma, ad esempio, atomica o nucleare.

(*) Nota presentata al 20 Congresso Intemazionale Tecnico Scientifico dello Spazio, Roma, 19-23 Giugno 1962. 
Orviamente, i diversi sistemi hanno ciaseuno vantagegi e svantaggi, dovuti principalnente alla variazione del rapporto peso-potenza, potenza che a sua volta è valiabile nella sua entità con la durata e la natura del viaggio spaziale; è da notare che il rapporto peso/potenza per gli intpianti spaziali è condizionato dalla potenza, quando si tratti di sistemi sfruttanti energia presente nello spazio, e dallenergia quando siano sistemi sfruttanti sorgenti di energia trasportata da terra.

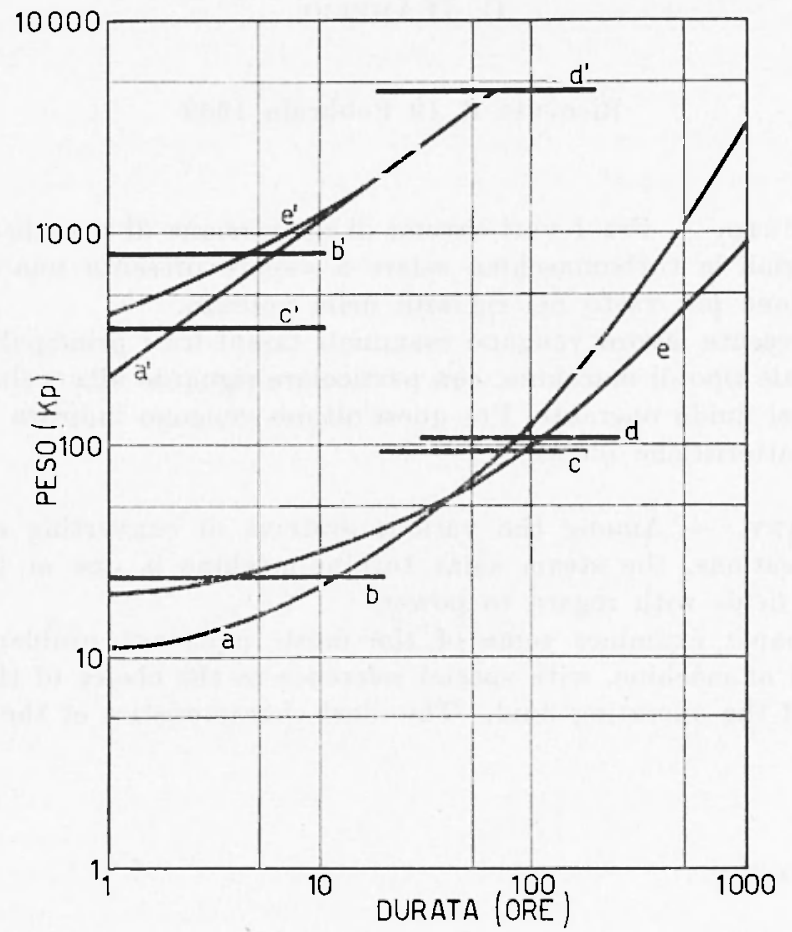

Figura 1

$a=$ Energia Chimica - Ciclo aperto - $1 \mathrm{~kW}$.

$a^{\prime}=$ Energia Chimica - Ciclo aperto - $100 \mathrm{~kW}$.

$b=$ Turbomacchina solare - Ciclo chiuso $-1 \mathrm{~kW}$.

$b^{\prime}=$ Turbomacchina solare - Ciclo chiuso - $100 \mathrm{~kW}$.

$c=$ Energia nucleare - Senza schermo - $1 \mathrm{~kW}$.

$e^{\prime}=$ Energia mucleare - Senza schermo - $100 \mathrm{~kW}$.

$d=$ Celle solari - $1 \mathrm{~kW}$.

$d^{\prime}=$ Celle solari $-100 \mathrm{~kW}$.

$e=$ Energia chimica - Ciclo chiuso -- $1 \mathrm{~kW}$.

$e^{\prime}=$ Energia chimica - Ciclo chinso - $100 \mathrm{~kW}$.

Le potenze richieste - trascurando nella presente analisi quella necessaria ai fini propulsivi, e considerando solo la potenza cosiddetta 
ausiliaria, rioè quella richiesta per la strumentazione e l'esercizio variano da qualche $W$ a diversi $\mathrm{kW}$, in relazione alla durata ed alla natura della missione spaziale. Pertanto, in funzione di tale durata e natura, vi sarà una determinata richiesta di potenza; poichè inoltre il peso del sistema di conversione è, come detto, funzione della potenza o dell'energia richiesta, è possibile ricavare un diagramma del peso dei diversi sistemi di conversione, per una determinata potenza, in funzione della durata della impresa (Fig. 1).

Quando si tratti invece di sistemi in cui il rapporto peso/potenza è condizionato solo dalla potenza, è possibile ricavare un diagramma (Fig. 2) del peso in funzione della potenza.

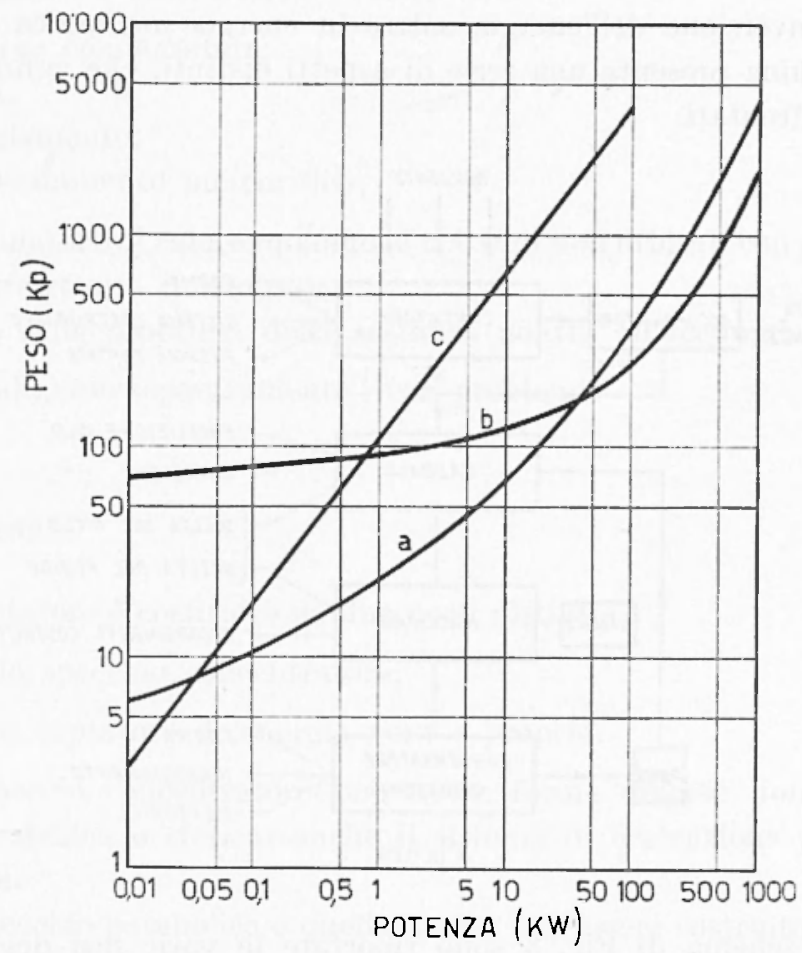

Figura 2

a) Turbomacchina solare;

b) Macchina nucleare a ciclo chiuso;

c) Batterie solari.

E possibile osservare dall'analisi di questi diagrammi (1) come sia conveniente da un punto di vista rapporto peso/potenza la conversione dellenergia solare in energia direttamente utilizzabile mediante turbo- 
macechina a vapore, quando la natura a la durata della missione spaziale richiedano potenze alusiliarie comprese nel campo fat i 30 Watt ed i $30 \mathrm{~kW}$.

Ci si interesserà, in questa breve esposizione, dei problemi relativi alla conversione dell'energia solare in energia meceanica nello spazio, mediante turbomacehina a rapose, eon particolare riguardo alla seelta del ciclo termico e del fluido più adatto, indicando infine quali dorrebbero essere le caratteristiche irladi del fluido.

II. PROMI,FIA.

La conversione dellenergia solare in energia meceanica mediante turbomacehina presenta ma serie di aspetti distinti, che ranno separatamente affontati.

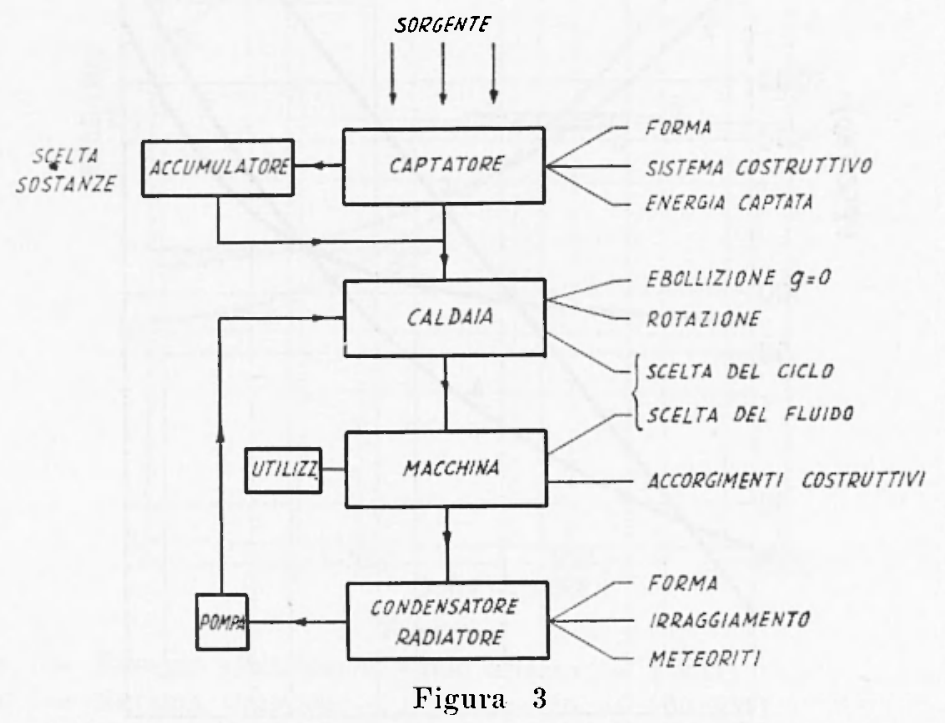

Sello schema di Fig. 3 somo riportate le vario fasi del processo, dalla captazione dellenerogia (captatore-caldaia), alla ntilizzazione dellenergia trasformata in energia mecoanica.

Follo schema stesso sono riportati anche quelli che sono alcuni fra i maggiori problemi, reativi alle rarie fasi cles processo, e cioc lispettivamente:

1) ('ap)tatole:

forma del aptatore, 
sistema costruttivo,

energia captata;

b) Calclaial:

fenomeni di ebollizione in camulo gralvitazionale nullo, rotazione della caldaia;

c) Caldaia-Macohina:

sceetar del ciclo,

sceelta del fluido operante;

d) Macehina:

accolgimenti costruttivi;

e) Radiatore condensatore:

forma,

imaggiamento,

bombardamento meteoritico;

f) Accumulatore di calore (quando si tratti cli voli orbitali con parte della traliettoria in ombla):

scelta delle propriceta della sostanza adatta all'alcemmulazione.

Si analizzano separatamente i vari problemi.

\section{IL OAPTATORE.}

Il (anptatore ì rostituito da due parti distinte:

1) lo specechio concentratore;

b) il captatore di energial rero e properio.

Lo speceio concentratore puo avere forme diverse, fondamentalmente parabolica o sferica; anche il sistema di costruzione può essere di valli tipi.

Lo specerio parabolico o quello sferico può essere costruito a sezioni, montate fra loro, con la possibilita di aprirsi automaticamente o a comando.

Lal superficie riflettente, gemeralmente con coefficienti di riflessioni dell'ordine di 0,98 , puó essere metallica o con depositi metallici su superfici particolari.

Un tale sistema concentratore, mentre fornisce ottimi risultati per quanto riguarda la captazione dellenergia, presenta lo srantaggio del peso relativamente elevato, laggiungendo valori intorno a $2 \mathrm{~K}$ / $/ \mathrm{kW}$. 
Un ulteriore tipo di specehio, di concezione più semplice è quello rostituito da un pallone sferico di plastica gonfiato, in cui una calotta è resa riflettente mediante depositi sottili di materiali metallici.

In tali tipi di specchi si ha il vantaggio del minimo peso per $\mathrm{kW}$ (cirea $0,01 \mathrm{Kp} / \mathrm{kW}$ ), ma naseono degli inconvenienti di altra natura. non facilmente eliminabili.

La superficie del pallone avà una determinata traspanrenza, necessariamente non totale, e pertanto non tutto il flusso d'energia incirlente su essa raggiungera lo specerhio.

Ancola, e problema questo più grave, il pallone è soggetto, come le altre parti della macchina, al bombardamento meteoritico, che provoca la foratura, o persino il laceramento, del sottile strato di plastica. Questo fenomeno conduce al conseguente cambio di forma del pallone, e dello specehio, per effetto delle tensioni superficiali della plastica non più equilibrata della differenza di pressione fra l'interno e l'esterno del pallone.

Comunque, a parte tali considerazioni di cantertere pratico, è forse opportumo richiamare l'attenzione sul sistema di roncentrazione.

Esiste una limitazione fondamentale per quanto riguarda la possibilità di concentrazione dell'energia da uno specechio parabolico o sferico che sia. Questa limitazione è data dal fatto che i ragoi solari incidenti non sono paralleli, ma convergenti con un angolo pari al diametro apparente del disco solare cioè al rapporto fra diametro del sole e la distanza del sole dallo specehio concentratore.

Orviamente, detto $\beta$ tale angolo la superficie riflettente rifletterà, in ogni punto, verso il collettore, un cono ri raggi con angolo all'apice $\beta$, rendendo impossibile la concentrazione di tutta l'energia in un punto (Fig. 4).

Mentre nel caso di reicoli orbitali tale angolo resta pressocehé (*ostante, variando poco la distanza del veicolo dal sole rispetto alla sua distanza media, nel aso di missioni spaziali non orbitali la variazione di $\beta$ può assumere una certa importanza, poiché la concentrazione dei raggi diminuisce a mano a mano che of aumenta.

E possibile dimostrare ed è ovvio dalla semplice osservazione della Fig. 4, rhe con mo specehio parabolico si riesce ad ottenere una concentrazione molto migliore che non arloperando mo specechio sferico.

I raggi solari vengono concentrati su un collettore che puo arere forma emisferica o piana; nel caso di collettore enisferico, la quantità di energia captata, a parità di specechio, risulterà maggiore. 
Lienergia cosi captata serve al riscaldamento ed alla evaporazione del fluido elastico nel collettore.

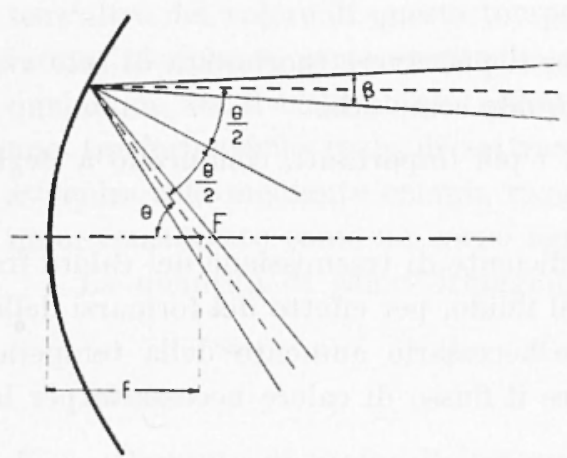

a

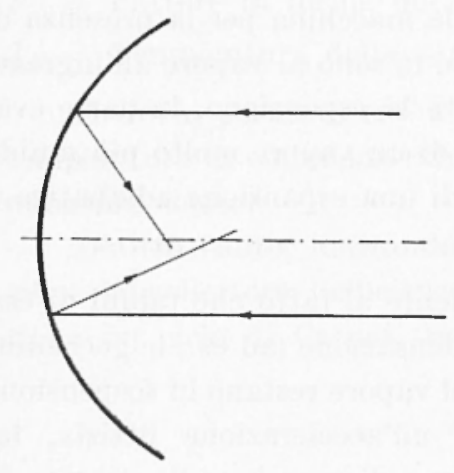

$b$

\section{Figura +}

a) riflessione su specchio parabolico;

b) riflessione su speechio $\mathrm{sfe}$ ricos.

\section{IAI CALDAH.}

Satumanente, nel veicolo spaziale l'aceelemazione, nellat palte del viaggio in cui non vi è spinta, sand nulla; come è noto, il fenomeno della ebollizione in campi gravitazionali nulli assume un aspetto alquanto diverso da quello normale.

Infatti, sperimentando su tale tipo di abollizione $\left({ }^{2}\right)$, somo stati riscontrati alcuni particolari fenomeni, che possono essere cosi riassunti:

a) Te bolle di vapore che si fomano in semo al liquido, non si allontanamo da esso formamelo, in mono col liquirlo stesso, ma schimma; 
b) Il flusso di vapore dalla caldaia al condensatore si riduce fortemente fino ad arrestarsi talvolta, con conseguente aumento della pressione della raldalia;

c) Quando il flusso ha luogo, si può avere fuoriuscita di solo vapore, della surdretta schinuma o di liquido come tale.

Questi fenomeni, che sono fra i più importanti, conducono a cleggli inconvenienti quali:

1. Una diminuzione del coefficiente di trasmissione del calore fra la parete interna del captatore ed il fluido, per effetto del formarsi della sehiuma suddetta, con conseguente necessario ammento della temperatura delle pareti stesse per ottenere il flusso di calore necessario per la potenza richiesta alla macechina.

2. I damni relativi al punto b) eioè alla riduzione del flusso di vapore ed aumento della pressione della caldatia sono di conseguenza evidenti.

3. Infine, la fuoriuscita di schiuma dalla caldaia, oltre ad alterare il fenomeno dell'espansione nell'ugello della macehina per la presenza di gocee di liquirlo in condizioni di saturazione in seno al vapore all'ingresso dellegello (gocee che ovriamente, durante la espansione, in parte evaporano), comporta l'ingresso in macchina di un vapore molto più umido di quanto non si sarebbe avuto nel caso di una espansione adiabatica a partire da rapor saturo.

Questa serie di inconvenienti, mitamente al fatto che taluni di essi si ritrovano anche nel fenomeno della condensazione (ad es., le goccioline di liquido derivanti dalla condensazione del vapore restano in sospensione nel condensatore), consigliano di creare un'aceelerazione fittizia, facendo ruotare, in un impianto di conversione di energia nello spazio, sia il collettore che il raliatore-condensatore.

Ferma restando quindi la soluzione, scelta peraltro da diversi antori proponenti tali impianti, di rendere sia il collettore che il radiatore rotanti, varrà osservare, a questo punto, quale sono a nostro arviso, il ciclo termico ed il fluido più conrenienti per questo tipo di macehina.

\section{IL FLU1DO ED IL CICLO TERMICO.}

In una torbomacehina spaziale, alimentata da energia solare con concent razione di raggi, la scelta del fluido operante è subordinata essenzialmente al peso dell'insieme per unità di potenza e al rendimento del riclo. 
Quest ultimo nel caso di macehina a vapore con temperature superiori al di sotto alla temperatura critica del fluido evolvente è funzione senz'altro del valore di questa temperatura superiore e di quella dell'isoterma inferiore (a parte eventuali surriscaldamenti). E da osservare che qualunque sia il ciclo chiuso adoperato dalla macechina, esso presenta una trasformazione reale di sottrazione di calore, sottrazione che può arveniese solo mediante energia raggiante da un radiatore verso il cielo nero, considerato come un corpo nero alla temperatuma di $0^{\circ} \mathrm{K}$.

La quantità di calore irraggiato dal radiatore sarà:

$$
Q=K \varepsilon A F T_{R}^{4}
$$

Dore:

$$
\begin{aligned}
& \boldsymbol{K}=\text { Costante di Stefan-Boltzmam } \\
& \varepsilon=\text { Coefficiente di emissività del radiatore } \\
& A=\text { Superficie totale radiante } \\
& F=\text { Fattore di forma del radiatore } \\
& T_{R}=\text { Temperatura della smperficie radiante. }
\end{aligned}
$$

E ovio pertanto che a parita di forma del radiatore, una elevata temperatura di emissione riduce la superfice radiante e quindi il peso del radiatore stesso.

D'altro canto, indicando con $T_{C}$ la temperatura assoluta di captazione del collettore, l'efficienza del ciclo, considerato in prima approssimazione 1111 ciclo di Carnot, risulta espressal da

$$
\eta=\frac{T_{C}-T_{R}}{T_{C}}
$$

Ia temperatuma massima del ciclo $T_{C}$ risulta limitata peró dalle (alatteristiche tecologiche del generatore.

Una bassa temperatura di radiazione ammenta le dimensioni del radiatore; esiste, ovviamente, per una determinata potenza del complesso, un "optimum " per il rapporto fla la temperatura del collettore e del radiatore che rendono minimo il peso del complesso, per un determinato valore del rapporto fra il peso dell'mità di superficie dello specechio e quello dell'unità di superficie del radiatore, assunto come parametro. Fissato tale parametro, il rapporto ottimo fra la temperatura massima e minima del ciclo, e cioè del collettore e del radiatore, puó essere ricavato (on lausilio di particolari diagrammi (').

Orviamente, all'aumentare dell'isoterma superiore si ha malliduzione in peso del complesso, a parità di rendimento, ovvero un rendi- 
mento maggiore a parità di peso, il che si traduce romunque in un minor peso dell'insieme a parità di potenza.

In un progetto recente $\left({ }^{3}\right)$, utilizzante come fluido operante vapori di Rubidio, fissata l'isoterma superiore a $6500 \mathrm{C}$, si do ottenuta una isoterma inferiore pari a $3500 \mathrm{C}$, con $\mathrm{m}$ rapporto fra i pesi per unità di superficie del captatore a dello specechio pari a 0,4 circa.

Tuttavia, nel ciclo in discussione, qualora in prima applossimazione si facesse espandere isentropicamente il vapore di Rubiclio dalle condizioni di rapore saturo e sececo a $6500^{\circ} \mathrm{C}$ fino alla pressione corrispondente a 350 oc, si arrebbe un titolo di vapore praticamente inaccettabile per l'ul-

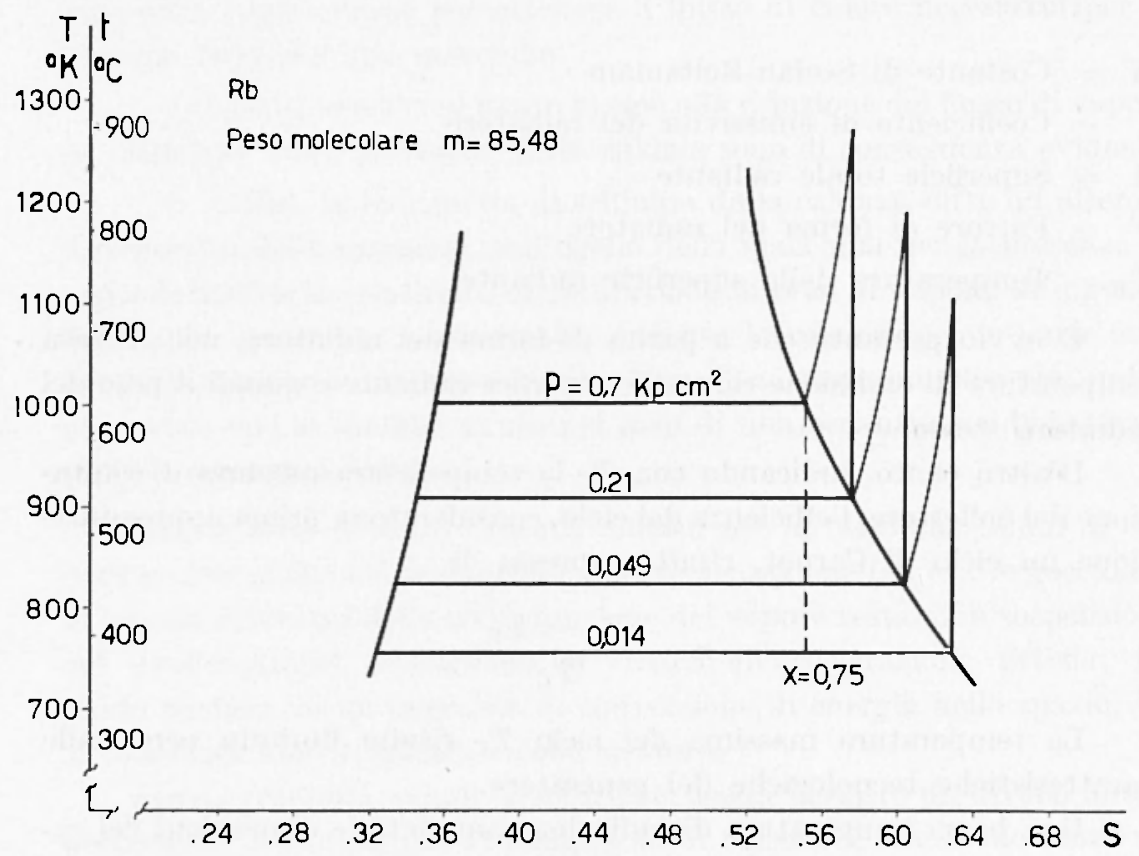

Figura 5 - Ciclo termico a vapore di rubirio

tima girante, $x=0,75$ (Fig. 5) dovendosi pertanto ricorrere a tre surriscaldamenti, con temperatuma massima intorno ai 1000 oO.

it ben evidente che in un impianto del genere il surriscaldamento risulta molto romplicato sia per quanto riguarda la diminuita semplicita dell'impianto (introduzione dei surriscaldatori, separazioni della mac(hina in più corpi) sia per l'orvio aumento di peso, che può essere anche noterole, dellintiero impianto. 
Un'ulteriole complicazione che sarà analizzata nel paragrafo relativo alla accumulazione, nell'adozione di ciceli a surriscaldamento, si ha nel (aso di voli orbitali, nei quali è necessaria un'acemunlazione di energia per il periodo in eui il reicolo pereorese il tratto di orbita in ombra.

A nostro avriso quindi, il cicelo termice più adatto per tale tipo di impianto risulta quello di Rankine senza surriscaldamenti, con elevate temperature per l'isoterma superiore al fine di ammentare il coefficiente economico del ciclo stesso. Ma l'elevarsi dell'isoterma di evapolazione rende notevolmente difficile la sceelta del fluido in quanto ad esso si richiede di arese alta temperatura critica e pressioni di vapore saturo particolarmente basse alla tempelatuma superioge onde non essere costretti a ricorrere a generatori di vapore particolamente robusti e quindi di peso inammissibili per simili appanati.

Ancora, necessità contrastante con la prima, la tensione di vapore saturo alla isoterma inferiore non deve essere molto bassa, pere evitare il fortissimo aumento di rolume specifico del vapore.

Contempolancamente si richiede al fluido un adeguato valore della pressione di dissociazione alle temperature elevate, per ovviale alle note perdite di occultamento di calore dovute appunto a tale fenomemo. Questa condizione faubbe indirizzare la scelta relso sostanze semplici (elementi) possibilmente a molecola monoatomical.

Di contro, la molecola costituita da un numero di atomi ridotto porta generalmente al calatteristico andamento della curva limite supeliore che risulta ad entropia crescente al diminuire della temperatura di satulazione.

Cio porta a titoli di vapore particolarmente bassi, con i noti inconrenienti.

E stato osservato che, in prima approssimazione, la pendenza della curva limite superiore risulta dipendente dal numero degli atomi nella molecola, indipendentemente dal peso regli stessi $\left({ }^{4}\right)$; tale curva limite superiore presenta una pendenza sempre minore al crescere del numero degli atomi, fino ad assumere un andamento ad entropia costante alle varie temperature (almeno nell'intervallo di temperature calatteristiche per tali impianti) ed infine addirittura, per molecole costituite da un numero notevole di atomi, un andamento ad entropia cerescente con la temperatuma (Fig. 6).

E bene chiaro ehe, utilizzando per le ragioni suesposte un ciclo di Rankine senza surriscaldamenti, per ottenere un buon titolo di vapore allo scarico a partire da vapor saturo, si doverebe adoperare un fluido con una pendenza della couva linite superioge pressoché nulla portando 
i fluidi con pendenza negativa a valori del titolo geneialmente troppo bassi ed i fluidi con pendenza positiva della curva limite superiore addirittura a rapori surriscaldati, con evidente abbassamento del rendimento del ciclo, qualora non si adoperasse uno scambiatore di calore.

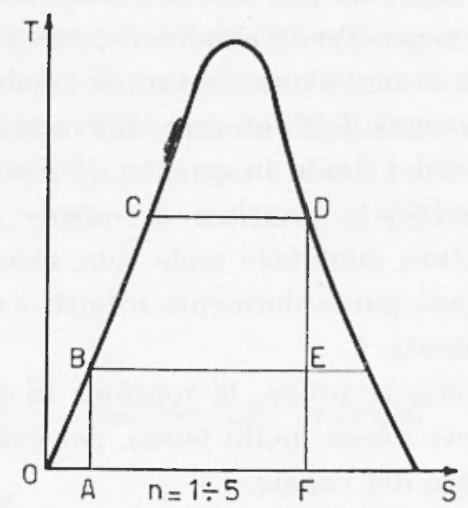

a

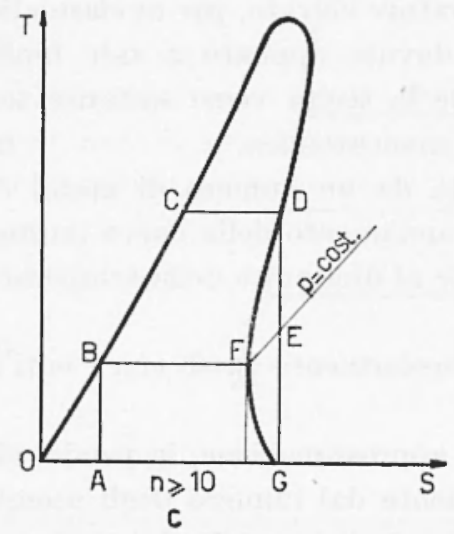

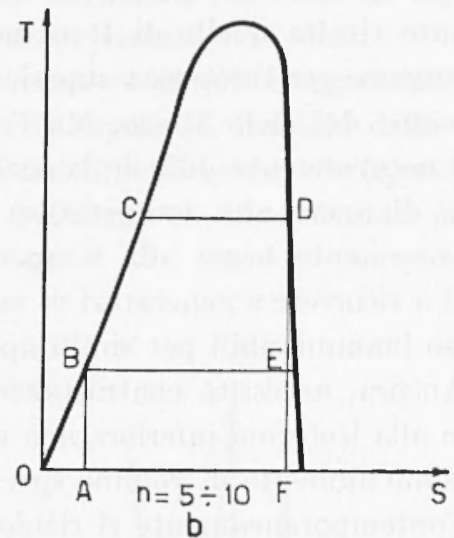

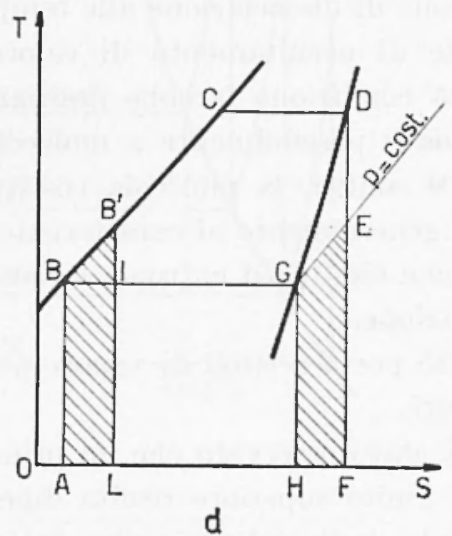

$n=$ iumero atom: della molecola

Figura 6 - Curve limiti di fluidi in relazione al numero di atomi della molecola.

Infatti istato dimostrato $\left(^{5}\right)$ che quando il ciclo assmme la forma ripoltata in Fig. 6d, e la quantità di calore lapperesentata dall'area tratteggiata viene riutilizzata col riscaldamento del liquido da $B$ a $B^{\prime}$, il rendimento del ciclo resta praticamente uguale a quello del ciclo di Rankine fäa le stesse isoterme. 
Ne consegulue, però, la necessaria presenza dello scambiatore di calore, con tutti gli inconvenienti relativi a questo delicato organo, primo fra tutti il peso.

Pertanto, ore mai si rolesse adoperale il ciclo di Rankine senza surriscaldamenti (a nostro arviso, come detto, il più conveniente), bisognerebbe rirolgersi a fluidi in cui la pendenza della curva limite superiore fosse pressoché nulli.

Volendo seguire questo indirizzo, si roverebero abbandonale i rorpi semplici oggi proposti quali il Mercurio, il Rubirlio, il Sodio ece. per rivolgersi a sostanze con molecole complesse che però possano mantenere lo innanzi esposte caratteristiche, come la stabilita alle alte temperature e la tensione di vapore non elevata alle temperature stesse.

Sostanze con molecole costituite da molti atomi e quindi generalmente con alto peso molecolare presentano inoltre l'innegabile pregio rli permettere un efflusso di vapore a parità di isoterme estreme, a velocità ridotte potendosi considerare con sufficiente approssimazione tale velocità proporzionale alla radice quadrata dello inverso del peso molecolare. a parità di $\gamma(*)$.

Da questo punto rli vista, il Mercurio si presenterebbe particolalmente adatto in quanto pur presentando, in accordo col fatto di aver una molecola monoatomica, un titolo allo scarico abbastanza basso, presenta un elevato valore del peso molecolare, $m=200,61$, cosa che permette di impiegare turbine con cunero ridottissimo di elementi.

In relazione al peso molecolare il Difenile, con $m=15 \pm$ potrebbe avere un notevole interesse, mentre il Rubirlio, con $m=85,48$ presenta già relocità di efflusso notevoli e tali da richiedere, nel campo considerato, una turbina a tre starli pelaltro, come si è visto, con riscaldamento intermerlio.

Noterole importanza assume la rensità rel liquido alla temperatura del comdensatore nel caso di macehine con caldaia e condensatori rotanti

$\left({ }^{*}\right)$ Risulta infatti $\left({ }^{6}\right)$, ammettendo in prima approssinazione la

$$
\begin{gathered}
\mu e=R T \\
c=1,985 \frac{\gamma}{\gamma-1} / \frac{\Gamma T}{m}
\end{gathered}
$$

dove:

m. - peso molecolare del fluido

$\Delta T=$ differenza fra le temperature estreme

$\gamma=\frac{e_{p}}{e_{v}}$

c - velocità di efflusso del vapore in 31 sec. 
come la maggior parte dei progetti di turbomacehine spaziali, rotazione pressoché necessallia come visto in precedenza, principalmente per simulare il campo gravitazionale inesistente, e semplificare in tal modo $\mathrm{i}$ fenomeni di ebollizione e di condensazione. Una clevata densità pemette con una certa facilita il ritorno del liquido dal condensatore alla caldaia semza bisogno di pompa di alimento ma utilizzando la semplice azione centrifuga, evitando al riguardo qualsiasi soluzione di continuità che potrebbe comparire per l'azionamento della pompa di alimento necessariamente dallestemo in vista dello speciale fluido.

Il fluido, oltre le accennate caratteristiche, deve ancora essere compatibile con i metalli impiegabili nella costruzione, possedere elevato rapporto rily ed altri requisiti secondari.

IA MACCHIXA.

Sulla è da dire in questa sede, nei riguardi della macehina non presentando essa, da 110 punto di vista non prettamente costruttivo, alcuma calatteristica peculiare.

\section{ACCUMUlatToRE DI ENERGiA.}

Sel caso di veicoli orbitali, la traiettolia dellinsieme attrarerserà periodicamente zone dello spazio direttamente investite dai raggi solari a zone d'ombial della ter'en.

Nasce pertanto il problema della acemmulazione dell'eneregia, per l"utilizzo nei periodi in cui il satellite viaggi in zona d'ombra.

Trascurando i tradizionali sistemi di accumulazione sotto forma clettrica, che per gmandi potenze risultano assolutamente inadatti allo scopo sia per il loro elevatissimo peso, sia per la loro delicatezza, il problema è stato affrontato da un punto di vista acemmulazione dell'energia, sfruttando il passaggio dallo stato solido allo stato liquido e viceveresa di talume particolari sostanze nel campo di temperatura richiesto.

Uno studio sulle proprietà chimico-fisiche degri idruri metallici ha messo in evidenza gli elevati calori e temperature di fusione di questi composti, e quindi la loro capacità a servire da accumulatori di calore con temperatura intomo ai $6000 \mathrm{C}$.

Importante, per la scelta della sostanza adatta, risulta la curva della pressione di dissociazione con la temperatura. Infatti è necessario 
evitare la dissociazione dell'idruro in idrogeno e metallo, che porterebbe alla variazione delle caratteristiche termofisiche del composto ed all'occultamento di parte dell'energia somministrata, non sfruttabile a temperature superiori a quelle di dissociazione.

Per evitare tale perdita, la pressione nell'acemmulatore di energia deve essere superiore a quella di dissociazione, alle varie temperature: appare quindi ovvia la convenienza ad adoperare sostanze con basse pressioni di dissoriazione.

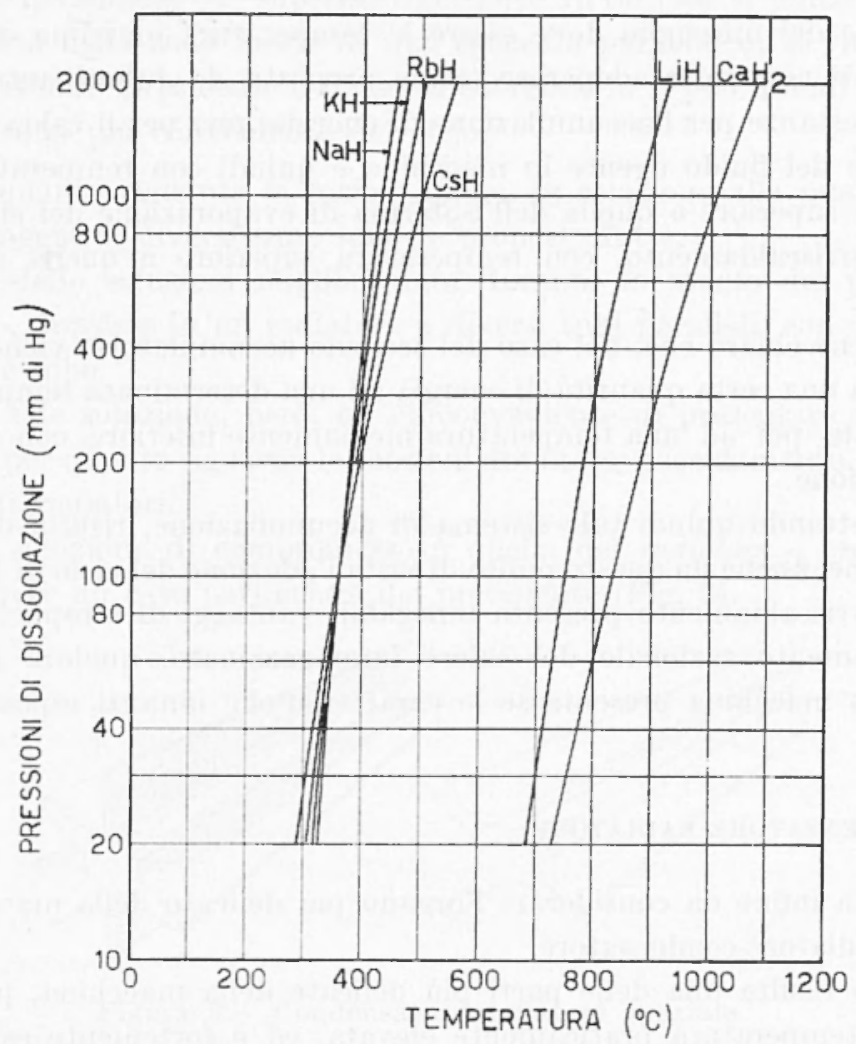

Figura 7 - Pressioni di dissociazione in funzione della temperatura di alemi idruri.

Sella Fig. i sono riportate appunto talume di queste curve relative ad un certo numero di idruri, dalle quali si puo osservare come gli idruri di Li e Ca presentino i più bassi valori della pressione di dissociazione a determinate temperature, e siano quindi i joiu facilmente ntilizzabili $\left.{ }^{7}\right)$. 
Sel recente progetto di eui precedentemente aceenmato, is stato proposto $m$ miscuglio formato dalla miscela di irlruro di litio e litio metallico, nelle rispettive percentuali di $95 \%$ e $5 \%$.

Un tale miscuglio, che ha mo elevatissimo calore di fusione per $\mathrm{kg}$ (cirea 600 heal/kp) presenta il vantaggio di non aver apprezzabili variazioni di volume fra lo stato solido a quello liquido, cosi da non generare, nell'interno dei condotti di scambio di callore delle sollecitazioni inaceettabili.

Ovviamente, nel caso del cirlo con sumiscaldamento, la temperatura di fusione del miscuglio deve essere la temperatura massima del ciclo, a meno di non voler adoperare (come proposto da taluni autori) due diverse sostanze per l'aremulazione di energia, una per il calore di evaporazione del fluido agente in macchina e quindi con temperatura leggermente superiore a quella dell'isoterma di evaporazione del ciclo, unla per il surriscaldamento, con temperatura superione a quella massima del riclo.

Fे bene chiaro che, nel caso del secondo accumulatore, viene immagazzinata una certa quantità di energia ad una determinata temperatura, a sfruttata poi ad una temperatuma mediamente inferiore, con evidente degradazione.

Areettando quindi tale sistema di arecumulazione, risulta di conseguenza che, anche ra questo punto di vista l'adozione del ciclo di Rankine senzal surriscaldamento presenta innegabili vantaggi di semplicità, peso e sfruttamento razionale del calore immagazzinato, qualora il fluido agente in macehina presentasse le caratteristiche innanzi esposte.

\section{IL COXIEXSITORE-RIDIATORE.}

Resta infine da considerane loorgano più delicato della macehina, e riò̀ il radiatore-condensatore.

Esso risulta una delle parti più delicate della macehina, perché si trova a temperatura praticamente alevata, ed i fortemente esposto al bombardamento meteoritico.

Il radiatore può essere di forme diverse, dovendo tener conto:

a) della resistenza al suddetto bombardamento meteoritico;

b) della massima (alpacità di irraggiamento, o, ciò ('he ì lo stesso di un elevato valore del fattore di forma.

Per quanto riguarda la prima rlifficoltà, le soluzioni proposte sono di diversa natura, sfruttanti un doppio principio: 
1) rendere le diverse zone del radiatore-condensatore stagne; ciò si può ottenere creando diversi cireniti di fluido, indipendenti. Qualora il condensatore-radiatore fosse colpito da un meteorite, la circolazione della zona colpita può essere interrotta automaticamente o, nel caso di reicolo abitato, dall'equipaggio. Questo comporta un pressoché necessario sovadimensionamento del rabliatore stesso, pur senzal superare determinati limiti.

2) esporre il radiatore il meno possibile al bombardamento meteoritico, riparandolo con superfici riflettenti. In tal caso il maliatore può essere posto nella zona focale di uno sperechio parabolico; si viene cosi anche a rendere direzionale il flusso di enereria e lo si puo quindi dirigere verso la zona piǹ conveniente del cielo.

Per quanto riguarda la forma, infine, in relazione alla quantità di calore irraggiato, diversissime sono le proposte fatte.

Ena delle soluzioni migliori nata durante lo studio del progetto "Elettra ", consiste in un radiatore a diversi tubi paralleli, con riflettori fira tubo e tubo.

Una tale soluzione, però, ha l'inconveniente di presentare noteroli problemi per quanto riguarda le tensioni dovute a riscaldamento, almeno per grandi randiatori.

Ena soluzione di compromesso è quella del radiatore a croce, che risulta essere un caso particolare del precedente (Fig. s).

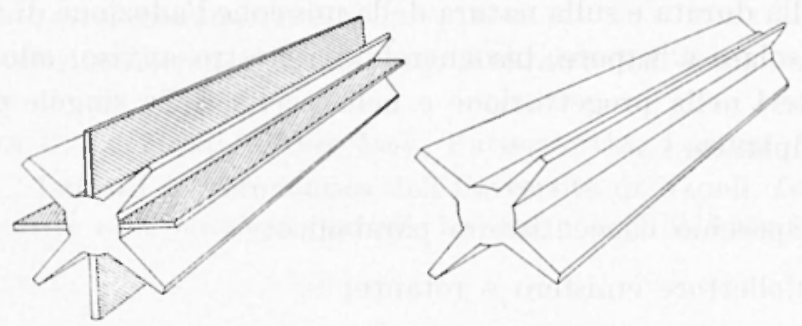

Figura 8 - Condensatore-radiatore spaziale

Per evitare l'iraggiamento reciproco fra elemento ed elemento, renerono interposti dei riflettori.

E possibile dimostrare che il lapporto fä il calore irraggiato da 101 condensatore-maliatore del tipo a croce senza i riflettori e lo stesso con i riflettori risulta essere:

$$
\alpha=\frac{1-F}{1-F}
$$


dove

$F=$ fattore geometrico di forma

$\varepsilon=$ coefficiente di emissivita.

Normalmente, il valore di a lisulta di poco inferiore all'uniti.

Eliminando i riflettori, ed ammentando la superficie irraggiante (com allungamento del radiatore) così da riportare al valore iniziale la quantita di alole emessa, si ha un aumento del peso che risulta genemblumente essere inferiore al peso dei riflettori, in virtic del fatto che il fatto geometrico di forma $F$ varia ron l'allungamento. In tal modo il complesso risulta più lexgero, sia pure di ma piecola quantita, ma reptamente meno delicato.

Tale soluzione dovrebbe risultare, a nostro arviso, ma delle più conveniente dal punto di vista della forma in relazione alla quantita di (alore irmagriata.

\section{Conclesione.}

Ia tutto quanto esposto, si possono trarre le seguenti conclusioni:

Quando nella scelta di un sistema di conversione di energia nello spazio risulta più conveniente, in base a considerazioni sulla potenza richiesta, sulla durata e sulla natura della missione l'adozione di una turbomacehina solare a vapore, bisognerebbe, a nostro arriso, adottare i seguenti criteri nella progettazione e nella scelta delle singole parti costituenti l'inpianto:

a) Specchio roncentratore parabolico:

b) Collettore enisfero a rotante:

c) Ciclo di Rankine senza surriscaldamenti;

d) fluido presentante un noterole mumero di atomi nella molecola. temperatura critioa alta, elevata stabilità, pressioni di satumaione alla isoterma superiore non erecessive:

- e) Condensatore-radiatore rotante, con superfici ralianti a 900 .

Ena marehina progettata con tali caratteristiche dorrebbe, a nostro arviso, presentare le migliori caratteristiche in relazione ai fattori peso e sicurezza di funzionamento. 


\section{BHIBIOUGRAFIA}

(1) Zimmermans R. L.. ZWick E. B.. space rehicle pouer systems. "J. A RS \#, 29. 8 553-565. (1959).

$\left({ }^{2}\right)$ Lat Lloyd M. IIEdgepeth, Zero gravily boiling and condensing. "Progress in Astronautics and Rocketry m. 3, 593, (1961).

(3) MaCAULEY B. T., The derelopment of a $1.5 \mathrm{kll}$ (el) solar meeanical engine for space application and its relationship to the fabrication of similar engines for terrestrial usage. U.X. Conference on new sources of energy. Rome, 1961, E/Conf. 35/S/27.

(1) Gobsstex II., National Laboratory of Istael, slope of the salured anor line in a temperature entropy diagramm. Lanoro non pubblicato.

${ }^{(5)}$ 'Tabor 11. - Brosicki I.. Small Turbine for solar energy porer package. C.X. Conference on new sources of energy. Rome, 1961. Elconf. $35 / 5 / 54$.

$\left.{ }^{(}\right)$I)'A nelo I., Limpiego di rapore ad allo peso molecolare in pircole lubine. Ea. I.A.A.(T., Sapoli, (1935).

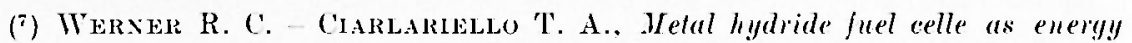
storage devices. IT.X. COnference on new sontes of energy. Rome, 1961. E/(Gonf. 35/Gen14

ed inoltre:

Bublock R. O., Fapor turbine for space power, da "Energy conversion for space ", 517, London (1961).

Handbook of Austronatical Enginering. MeGraww-Hill Book Company, ine. 1961.

S.METHenss (.J., Metals refence book. Butterworths, London 1962.

I)'Fun G., listituto di Aeronautica dell'Universita di Napoli, he potenze non propulsice nelle missioni spaziali. Lavoro non pubblicato. 


\title{
Precisazione "Sui risultati di alcune ricerche sulla serie gessoso-solfifera siciliana "
}

\author{
A. Frincaviglia
}

Ricevuto l:11 Aprile 1963

\begin{abstract}
Riassunto. - L'A. richiama alcuni dati di fatto che un anonimo recensore, commentando ma sua nota nel $n^{\circ} 34$ della rivista "Lo Zolfo ", ha, involontarianente dimenticato di riferire, sicché la nota predetta pnò essere interpretata come un attacco all Ente Zolfi Italiani, anziché alla Compagnie Gencrale di Géophysique, secondo lo scopo dell $\mathrm{A}$.
\end{abstract}

SUmary. - The Author recalls certain factual elements which had been involuntarly forgotten by the anonymous editor who commented a note of his in the issue No 34 of the magazine "Lo Zolfo". This inconveniency might have caused the note itself to be interpreted as an attack against the Ente Zolf Italiani, rather than the Compagnie Générale de Géplıysique, as the author had meant it.

Nella mia nota dal titolo sopra riportato, apparso sugli "Annali di Geofisica» (Francaviglia 1962), ho cercato di mettere in evidenza le cause del risultato negativo dell'indagine geofisica condotta dalla Compagnie Générale de Géophysique (C.G.G.) per incarico dell'Ente Zolfi Italiani (E.Z.I.) nelle zone di M. Stretto e Trabia-Tallarita nella Sicilia centrale.

Debbo dichiarare che lo scopo della mia nota è stato quello di far risaltare come la C.G.G., pur avendo avuto a disposizione i risultati dei rilevamenti geologici di dettaglio, i profili stratigrafici delle trivellazioni già eseguite nelle due zone, nonché tutti gli altri dati utili alla soluzione del problema proposto, abbia adottato un metodo di prospezione, che la sua lunga esperienza in materia doveva sconsigliare.

Come già è avvenuto in altra occasione (Francaviglia 1955, 1956), la C.G.C. non ha creduto opportuno controbattere le mie osservazioni, 
sia per assoluta mancanza di argomentazioni sceientifiche, sia perché i lisultati delle trivellazioni di controllo hanno contradlet to le sue deduzioni fatte in sede di relazione, e, infine, perché non poteva pubblicare le giustificazioni addotte per spiegare queste contraddizioni, che avrebbero certamente deposto a sfavore della C.G.G.

Ippolito a Manfredini $\mathbf{A}$. (1951) hamno scritto che molti risultati negativi delle ricerche geofisiche sono dovute al fatto rhe le Società di prospezione mirano, in prino luogo, ad aceaparrarsi un lavoro, approfittando della scarsa competenza che, in genere, i committenti hamno della materia, senza curarsi se le condizioni di giareitura del minerale cercato contrastino con le condizioni teoriche richieste per la corretta interpretazione dei risultati di campagna. Nel caso presente, l'E.Z.I. non aveva allora un proprio reparto geofisico o un consulente qualificato. Si romprende benissimo che abbia dato ascolto ai lapplesentanti di una Societa che si presentava con una esperienza ultratrentemnale.

Son comprendo, quindi, l'intervento dell'E.Z.I. in una polemica fin me e la C.G.G. - Non è mia intenzione di polemizzare con l'E.Z.I., ma sono costretto a rilevare alcune inesattezze nelle quali, certamente in buona fede, è incorso l'anonimo recensores.

I primi lavori di ricerea solfifera veri e propri somo stati iniziati dall'E.Z.I. nel 1949 con lo studio di dettaglio dei bacini di Trabia-Tallarita a di Lereara Frideli, per iniziativa del Prof. Gerbella allora Direttore teconico e Amministratore Delegato dell'E.Z.I., seguiti nel 1950 dai lavori nel bacino di Palma di Montechialro, studiato in precerlenza geologicamente e geofisicamente da me e Manfredini A. (Manfredini A. 1950, Francariglia 1951), e in quelli di Comitini-Aragona e Favara-Ciavalotta; nel 1951 dallo studio dei bacini compresi nel foglio 268 "Caltanisetta ".

Son è esatto dire ('he io sono "stato uno dei geologi (he ha aruto incarico dall'Ente Zolfi di eseguire rilevamenti alla scala di $1: 25.000$ in varie zone. Rilevamenti (he sono serviti anche per agoiornare la Canta Geologica della Sicilia ". Ciò è avvenuto soltanto dopo la creazione del Centro di Terrapelata a Caltanisetta.

Nella mia nota ho dato atto allE.Z.I. di non essersi mai contentato del parere di un solo geologo, e di essere ricorso all'indagine geofisica nei casi controversi. Ho, invece, rimproverato, e ancora ma volta rimprovero, alla C.G.G. di non arer tanto conto di quanto era già stato pubblicato, di quanto avevo scritto nelle mie relazioni all'E.Z.I., della relazione" del greologo Jaoul della Compagnie de Mines de Huaron, e di avere, pertanto applicato un metodo dhe non poteva dare, e non ha dato, i risultati richiesti per le condizioni di giacitura della serie gressoso-solfifera. 
La migliore prova della bontà del mio contributo alla ricerea soltifera e della fondateza della mia eritica costruttiva d data dalle pietose giustificazioni fornite dalla C.G.G. per giustificare il risultato negativo del suo lavoro nel caso in esame, e dal non arere essa rilevato le mie critiche pubblicate in riviste di ampia diffusione.

Roma, Istituto Nazionale di Geofisica, Aprile 1963.

\section{BIBLIOGRAFIA}

Francavigia A., Risullali di aleune ricerche sulla serie gessoso solfifera siciliana. "Amali di geofisica ", XV, Roma, (1962).

- Un esempio di mancata collaborazione jra geologia e geofisica: Vindagine idrologica nel lemilorio di Calania. "Boll. Soce. Geol. 11. ", LXXIII, Romal, (1955).

- Ancora sulla ricesea idrologica nei dinlorni di Calania. "Boll. Sor. Geol. It. ", LXXV, Roma, (1956).

- La ricerea del minerale solfijero in Sicilia. "Riv. Min. Sic.n, 7. Palerno, (1951).

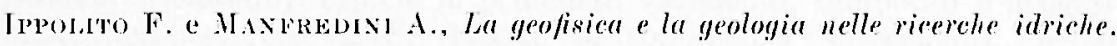
"LiAcqua ", 7-8, Roma, (1951).

MANFREDIN A.. Sludio geopisico della formazione gessoso-solfifera siciliana col melodo della resistivilù. "Boll. I'ft. Geol. d'It. ", LXX. Roma, (1950). 